\title{
Anticipated stimuli across skin
}

SIR - Our perceptions occur only after the information we receive from our senses has been heavily preprocessed. The degree to which cognitive influences shape this processing has been debated for many years. Sensory saltation is a robust class of illusions that has been described as a shift in the perceived location of a stimulus towards a rapidly delivered subsequent stimulus $^{1,2}$. Our studies show that stimuli evoked in $<200$-ms epochs are all mislocalized in sensory saltation. In the simplest two-stimulus form of the illusion, perceived stimulus locations are symmetrically shift-

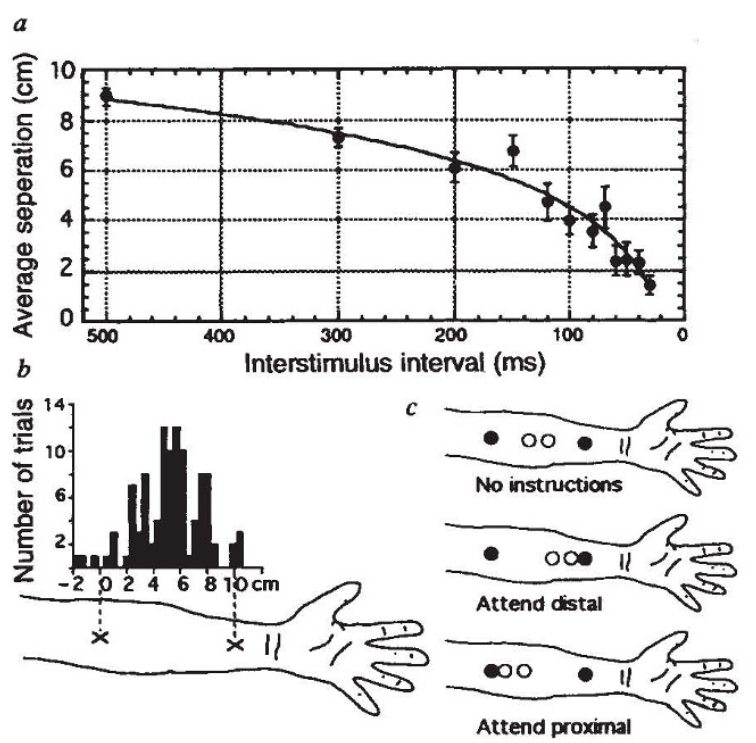

a, Perceived stimulus separation as a function of ISI. The forearm was hidden from the view of 13 naive subjects (Ss). After 10 repetitions of each ISI, Ss marked the locations of the two experimental taps with respect to the two locator taps. Bars are standard errors; $R=0.97$. $b$, Distribution of centre points of experimental tap pairs, with data pooled for 30-, 40-, 50and $60-\mathrm{ms}$ ISIs. Mean location: $5.26 \mathrm{~cm}$, s.d., $2.41 \mathrm{~cm}$. $c$, Representative examples of the perception of the two locator and the two experimental stimuli for an ISI of $60 \mathrm{~ms}$ without (top) or with specific instructions that influenced their directed attention. Filled circles, actual stimulus locations; open circles, perceived stimulus locations.

ed towards one another. A misunderstanding of the fundamental nature of the illusion apparently arose because powerful effects of directed attention on absolute stimulus localization were not recognized.

Geldard and colleagues discovered sensory saltation and described versions of it in the tactile, auditory and visual systems studying its tactile form as their principal model ${ }^{2}$. In their most basic tactile paradigm, a skin tap that served as a reference (the 'locator') was delivered $500 \mathrm{~ms}$ before the presentation of two experimental taps such that it did not influence the mislocalization. The first experimental tap was delivered at the locator site; the second 10 $\mathrm{cm}$ up or down the forearm. Geldard's subjects perceived the first experimental tap to be nearer to the second (the 'attractor', which was properly localized) as a linear an ISI of $100 \mathrm{~ms}$, the second tap was perbe halfway between the actual loci of the two experimental taps, that is, . $5 \mathrm{~cm}$.

- debated problem with Geldard's explanation of sensory saltation concerns a brain mechanism can make a if the following 'attractor' has not yet occurred $^{3,4}$. Our findings demonstrate that paradox arose from a misunderstanding of the illusion. Geldard and colleagues used trained subjects to reduce response variability. By training them, an important aspect of the illusion, a powerful biasing effect of directed attention (or expectation), was apparently circumvented.

We added a fourth stimulus at the second stimulus site as a second 'locator,' again well separated in time from the two experimental taps. In this simple symmetrical variation of Geldard's paradigm, naive subjects had no difficulty estimating the perceived separations between the two rapidly successive stimuli. However, although estimation of the absolute locations of the two stimuli on the forearm was quite variable, saltation stimuli were not perceived by naive subjects as one tap mislocalized towards the second, but rather, as taps located progressively closer to one another as the ISI was decreased ( $a$ in the figure). The centre points between the two perceived taps were distributed as a roughly gaussian function centred at a point midway between the two actual stimulus locations ( $b$ in the figure).

A main source of the substantial variability in locating the two events on the skin was made apparent when naive subjects were asked to concentrate on the proximal (or distal) region of the forearm. When so instructed, perceived separations between the two taps did not change, but the perceived skin locations of the two taps were dramatically shifted up or down the forearm in the attended direction ( $c$ in the figure). The average difference between the centres of the perceived locations when naive subjects were instructed to attend to the proximal versus the distal forearm was $3.1 \pm 0.47 \mathrm{~cm}$ (mean \pm s.e., $P<0.0001)$.
With practice, this powerful effect progressively strengthened, and could result in stimulus mislocalization exactly as Geldard described - or in an equivalently great opposite-direction mislocalization.

Thus, although the perceived separations of stimuli were determined by their physical separations and ISIs, the perceived locations of the stimulus pairs were largely determined by a subject's selective attention and/or expectation. We conclude that, in training, Geldard's subjects were effectively taught to concentrate on the 'attractor' location to reduce response variability.

A better understanding of the cutaneous saltation illusion gives us further insight into the fundamentals of tactile and haptic perception. The mechanisms that generate cutaneous saltation (and presumably related phenomena in vision and audition) operate as a smoothing process by which the perceived distances between two events occurring within a short time domain are generated by symmetric convergence, with locations powerfully biased by ongoing cognitive estimates of where those events are most likely to have occurred.

Michael P. Kilgard

Michael M. Merzenich

Keck Center for Integrative Neuroscience, University of California, San Francisco, California 94143, USA

1. Geldard, F.A. \& Sherrick, C.E. Science 178, 178-179 (1972).

. Geldard, F.A. Sensory Saltation (Erlbaum, New York, 1975).

3. Goodman, N. Ways of Worldmaking 74 (Hackett, Indianapolis, 1978)

4. Dennett, D. C. in Consciousness Explained 140-153 (Little, Brown, Boston, 1991).

\section{Improved green fluorescence}

SIR - The green fluorescent protein (GFP) from the jellyfish Aequorea victoria has attracted widespread interest since the demonstration ${ }^{1}$ that heterologous expression of the cloned gene ${ }^{2}$ can generate striking green fluorescence. Despite the tremendous potential of recombinant GFP as a marker for gene expression or cell lineage or as an in situ tag for fusion proteins $^{3}$, the wild-type protein from $A$. victoria has several significant deficiencies. Its excitation spectrum ( $a$ in the figure) shows peaks at both 396 and $475 \mathrm{~nm}$. The longer-wavelength excitation peak has the advantages of greater photostability ${ }^{1}$ and better matching to standard fluorescein filter sets, but is relatively low in amplitude. Considerable improvement should be possible, because a closely related protein ${ }^{4}$ from the sea pen Renilla reniformis has the same high quantum yield of emission $(0.7-0.8)$, yet shows only one absorbance and excitation peak with an extinction coefficient per monomer more than 10 times that of the longer- 